\title{
Das Strafvollzugsgesetz 1976 - Träumerei von gestern?
}

Burkhard Plemper

Ist das Strafvollzugsgesetz überholt und entspricht nicht mehr den Aufgaben des heutigen Strafvollzugs? Wieso gibt es so wenig Plätze für einen offenen Vollzug, scheitern Vollzugslockerungen an der chronischen Personalknappheit, wie umgehen mit der Überbelegung? Diese Fragen stellte Burkhard Plemper, Radio- und Fernsehjournalist, in seiner Sendung »Redezeit« auf NDR-Info am 21. August 2002 seinen Gästen, drei norddeutschen Justizministern: Prof. Dr. Christian Pfeiffer, SPD, damals noch Justizminister des Landes Niedersachsen, Anna Lütkes, Bündnis 90/Die Grünen, Justizministerin des Landes Schleswig Holstein, Dr. Roger Kusch, CDU, Justizsenator der Freien und Hansestadt Hamburg. Unter der Überschrift »Harter Knast oder Resozialisierung - was bringt mehr Sicherheit« ging es unter anderem um die Anpassung der Lebensverhältnisse in der Anstalt an das Leben draußen.

Neue Kriminalpolitik dokumentiert Auszüge aus dieser Diskussion. Die Textpassagen sind wörtlich wiedergegeben. Ergänzungen, die den Sinn deutlich machen, sind in (Klammern) gesetzt, Anmerkungen der Redaktion kursiv.

\section{Moderator:}

Es heißt im Strafvollzugsgesetz, der Regelvollzug, also die Regel des Vollzugs, ist der offene Vollzug. Ist das Ihrer Meinung nach so gerechtfertigt?

Kusch:

Das Strafvollzugsgesetz ist zu einer Zeit entstanden, in der man Hoffnungen in die Entwicklungsfähigkeit einzelner Menschen und ganzer gesellschaftlicher Gruppen gestellt hat, die sich aus heutiger Sicht als leicht utopisch oder jedenfalls weltfremd dargestellt haben. Der Glaube, die Mehrheit der Gefangenen sei für offenen Vollzug geeignet, ist bei der heutigen Klientel in allen sechzehn Bundesländern - das beschränkt sich nicht auf Hamburg - einfach nicht mehr wirklichkeitsentsprechend. Es gibt kein Bundesland, bei dem das Verhältnis zwischen offenem und geschlossenem Vollzug symbolisiert, daß die Leute, die den Vollzug gestalten, an dieses Märchen glauben. Und wir müssen mit diesem der heutigen Lebenswirklichkeit nicht mehr entsprechenden Gesetz leben und tun uns eigentlich auch gar nicht so schwer. Denn es geht nicht um statistische Vergleiche - wieviel Prozent haben (wir) im offenen und wieviel im geschlossenen (Vollzug) -, sondern um eine seriöse Analyse eines jeden einzelnen Falles. Und da kann es durchaus sein, daß in Stadtstaaten andere Prozentzahlen herauskommen als in einem Flächenland und im Süden an-

dere als im Norden. Denn der offene Vollzug birgt zusätzliche Gefahren für die Sicherheit der Bevölkerung, verglichen mit dem Geschlossenen. Ich hab's an einem Beispiel selber hautnah erlebt: Der alte Hamburger Senat (die rotgrüne Koalition, d. R.) hatte eine neue Vollzugsanstalt, die derzeit im Entstehen ist, als offene Vollzugsanstalt konzipiert. Der neue Senat hat festgestellt, daß für eine so hohe Zahl an offenen Plätzen gar kein Bedarf ist, und hat umkonzipiert, die offene in eine geschlossene Anstalt. Jedenfalls die Menschen, die in der Nähe dieser Anstalt wohnen, mit denen ich mich persönlich unterhalten habe, sind zum Teil nicht sehr begeistert, daß in ihrer Nähe eine Vollzugsanstalt gebaut wird, waren aber durchweg ohne Ausnahme sehr angetan davon, daß die Anstalt künftig eine Mauer haben wird.

Moderator:

Herr Pfeiffer, ist das gegen das Gesetz oder gegen den Sinn, gegen die Ziele des Strafvollzugsgesetzes, wenn jetzt verstärkt Haftplätze im geschlossenen Vollzug gebaut werden?

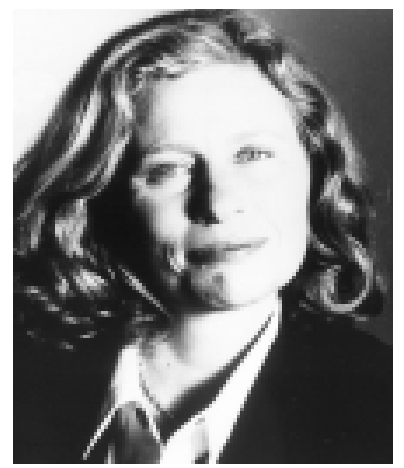

Justizministerin Anne Lütkes

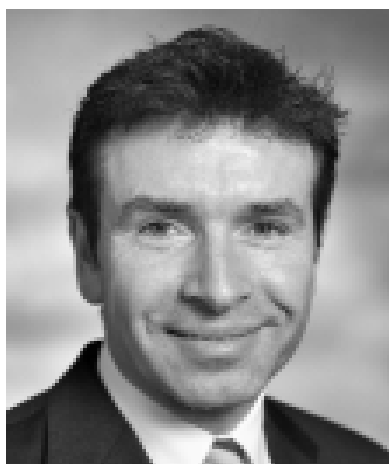

Justizsenator Roger Kusch
Pfeiffer:

Ich will nicht sagen »verstärkt«. In Hamburg mag es diese Rahmenbedingungen gegeben haben, daß man sich für diesen geschlossenen (Vollzug) entschieden hat. Aber, wie Herr Kusch sagte, das ist von Land zu Land unterschiedlich, hängt von der Struktur der Gefangenen ab. Also ich kann mit unseren Rahmenbedingungen gut leben und gebe Herrn Kusch recht: Die Mehrheit sitzt auch bei uns, die große Mehrheit, im geschlossenen Vollzug. Aber es ist keineswegs so, daß die Menschen, die in der Nähe eines offenen Vollzuges leben würden, Risiken ausgesetzt sind. Wir haben das ja genau überprüft: Was geschieht denn mit denen im offenen Vollzug? Da ist es durchaus so, daß der eine oder andere einen über'n Durst trinkt, mal nicht kommt. Aber wir haben keine Vorfälle von gravierenden Straftaten, die von Gefangenen während ihrer Vollzugszeit aus den Chancen heraus begangen werden, die sie im offenen Vollzug haben wegzulaufen oder dumme Dinge zu tun. Also, wir sortieren ja sorgfältig, prüfen genau, wem wir diese Möglichkeit des offenen Vollzugs geben. Es ist

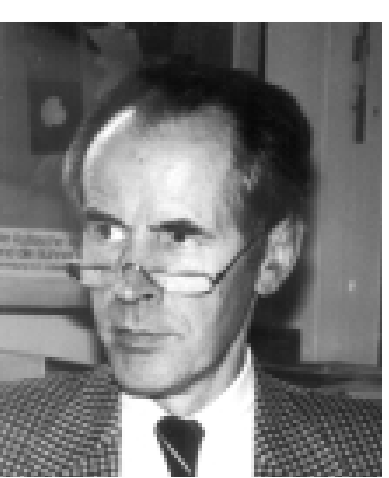

Justizminister a.D. Christian Pfeiffer 
meistens so, daß dies die Schlußphase einer längeren Haft ist, wo man sagt, in einem Vierteljahr, in einem halben Jahr ist er dann sowieso in Freiheit. Da macht es doch Sinn, gleitend den Übergang zu machen, damit er sich an normale Arbeitsbedingungen gewöhnt, damit das Rückfallrisiko reduziert wird. Das gelingt durch den offenen Vollzug. Von daher ist er eine notwendige, eine sinnvolle Ergänzung des Geschlossenen als Schlußphase einer längeren Haftzeit, um den Übergang risikofreier zu gestalten. Es ist im Interesse der Sicherheit der Bürger und funktioniert auch so ....

\section{Moderator:}

.... also als ein abgestuftes Konzept: Erst geschlossener Vollzug und dann offener....?

Pfeiffer:

.... wenn der Gefangene den Grund für diese Verlegung gibt, nicht automatisch, sondern dann, wenn wir sehen: Doch, hier können wir's riskieren. Und unsere Erfahrungen bestätigen das. Wir machen einen sehr verantwortungsbewußten, zurückhaltenden Gebrauch vom offenen Vollzug. Aber da, wo wir ihn einsetzen, ist er hilfreich für die Reintegration ins Arbeitsleben und für die Senkung von Rückfall.

$\ldots$

\section{Moderator:}

Ist das Strafvollzugsgesetz nach 25 Jahren Bestehen weltfremd, ideologisch?

Pfeiffer:

Nein, das ist es nicht. Es gibt einige wenige Vorschriften, wie die vorhin erwähnte über den offenen Vollzug, die gehen an der Wirklichkeit vorbei. Aber ansonsten sind wir mit dem Strafvollzugsgesetz zufrieden, weil es Spielräume eröffnet, im Rahmen des Machbaren beides zu garantieren; erstens den Schutz der Allgemeinheit - die Sicherheit und zweitens aber auch den Auftrag erteilt: Im Interesse dieser Sicherheit der Allgemeinheit vermeidet alles, was Schäden im Vollzug erhöht! Wirkt gegen schädliche Nebenwirkungen des Vollzuges! Und zweitens: bereitet diesen Menschen so auf die Entlassung vor, daß er nicht rückfällig wird! Beide Aufträge müssen erfüllt werden. Dazu gehört Behandlung, dazu gehört, Arbeit intern vernünftig zu organisieren, dazu gehört auch eine Vorbereitung der Entlassung nicht »Knall auf Fall « aus dem geschlossenen Vollzug ohne jede Vorbereitung nach draußen, sondern vorbereitende Maßnahmen, die diesen Rückfallrisiken entgegenwirken. Also ich kann mit diesem Gesetz bestens leben und sehe gar keinen Grund, es für schwach oder überholt oder sonst 'was zu erklären, und außerdem: Das Bundesverfassungsgericht setzt Maßstäbe wie beispielsweise kürzlich mit seinem Verbot, zwei Gefangene auf 7,6 Quadratmeter - gewisser- maßen in einem Wohn-Klo - zu halten. Da haben sie klar gesagt: Das verstößt gegen die Menschenwürde. Das setzt uns unter Druck und ist im Grunde genommen nur eine Konkretisierung der Maßstäbe, die das Strafvollzugsgesetz beinhaltet.

Kusch:

Das stimmt so nicht, Herr Pfeiffer, was Sie zu diesem Punkt gesagt haben: Das Strafvollzugsgesetz regelt, was diesen Punkt angeht, nämlich die Einzelunterbringung - das Bild, daß nur eine Einzelunterbringung den therapeutischen Zielen, die im Strafvollzugsgesetz niedergelegt sind, entspricht. Das Bundesverfassungsgericht hat sich mit einem völlig anderen Problem befasst, nämlich der Frage der Menschenwürde, wenn zwei Menschen in einem zu kleinen Raum ....

Pfeiffer:

Richtig!

Kusch:

.... mit sanitären Bedingungen ausgestattet gezwungen sind, voreinander eine offene Toilette $\mathrm{zu}$ benutzen.

Pfeiffer:

Das habe ich doch gerade gesagt!

Kusch:

Aber das ist nicht die Konkretisierung dessen, was im Strafvollzugsgesetz steht. Im Strafvollzugsgesetz steht die Einzelunterbringung, gegen die in Niedersachsen genauso verstoßen wird wie in allen anderen fünfzehn Bundesländern. Da kann ich sogar für Hamburg sagen, daß wir bei der Doppelbelegung, die auch in Hamburg unumgänglich ist, besser da stehen als viele andere Bundesländer.

Lütkes: .... murmelt ....

Kusch:

Doch, im Strafvollzugsgesetz steht: Doppelunterbringung ist unzulässig! Unabhängig von sanitären oder Menschenwürdefragen. Und meine Überlegung, die ich hier gerne wiederhole, ist: Es kann nicht richtig sein, daß man bei Soldaten, die irgendwo in einer Kaserne sind oder die irgendwo im Einsatz sind, überhaupt nicht auf die Idee kommt, zu diskutieren, ob eine Unterbringung von zehn Soldaten in einem Zelt gegen irgendwelche Menschenwürdeprobleme verstößt ...

Pfeiffer:

Aber Herr Kusch, das ist 'was völlig Anderes!
Kusch:

Nein!

Pfeiffer:

Die sind doch nicht 23 Stunden in einem Raum eingepfercht.

Kusch:

Welche Gefangenen sind denn dauerhaft in einem Raum ...

Pfeiffer:

Wenn sie keine Arbeit haben!

Lütkes:

Das lag dem Urteil des Bundesverfassungsgerichts zugrunde ....

Kusch:

Herr Pfeiffer, (das) aus dem Munde eines Justizministers überrascht mich, denn das ist ein Kampfbegriff, der der Realität in den Strafvollzugsanstalten - ich vermute nicht nur in Hamburg, sondern auch in Niedersachsen - widerspricht.

Pfeiffer:

Also 7,6 Quadratmeter auf zwei Menschen ....

Kusch:

Da sind wir doch über den Fall vom Verfassungsgericht einig, aber meine Äußerung, die ich hier wiederhole ist: An diesem Punkt ist das Strafvollzugsgesetz weltfremd!

....

\section{Moderator:}

Nun gibt es ja, Frau Lütkes, Kritiker, die fordern, der Strafvollzug solle auch der Sühne und der Sicherheit der Bevölkerung dienen. Ist das für Sie ein Widerspruch zur Resozialisierung?

Lütkes:

Nein, das sieht auch das Gesetz nicht als Widerspruch. Denn wir sind verpflichtet - alle, die wir hier sitzen, und der gesamte Strafvollzug -, sowohl den Resozialisierungsgedanken ernst $\mathrm{zu}$ nehmen und umzusetzen, aber auch dem Sicherheitsanspruch der Bevölkerung Genüge zu tun.

Moderator:

Was heißt das? 
Lütkes:

Das heißt - jetzt 'mal idealtypisch: Der Strafvollzug soll als Ergebnis in jedem Einzelfall haben, daß ein Mensch bei seiner Entlassung, zu der er ja hingeführt werden muß und vorbereitet sein soll, ein straffreies Leben führt und andere nicht mehr beeinträchtigt, nicht mehr gefährdet und nicht mehr verletzt. Das ist ja unser aller Ziel. Es ist ein bißchen der Glaube an das Gute im Menschen. Wir wissen aber, daß das nicht immer so durchgeführt werden kann, daß die Menschen nicht mehr rückfällig werden, weil: es gibt diese Fälle. Und insofern müssen wir uns natürlich auch der Aufgabe stellen, die Sicherheit durch Vollzug der Freiheitsstrafe zu garantieren, da kommen wir nicht drumrum.

\section{Moderator:}

Was heißt das denn konkret? Ist es: Die Sicherheit wollen Sie dadurch gewährleisten, daß Sie den Leuten ermöglichen, nach der Verbüßung der Haftzeit straffrei zu leben, oder heißt es, Sie wollen die Straftaten dadurch verhindern, daß Sie die Leute eingesperrt halten? In der Zeit können sie draußen keine Straftaten begehen.

Lütkes:

Also, das gute, idealtypisch wunderbarste ist: Es werden keine Straftaten mehr begangen.

\section{Moderator:}

Das ist das, was Herr Kusch für weltfremd hält ....

Lütkes:

Das hält er ..... und ich sage auch nicht, daß das hundertprozentig möglich ist. Das wäre wahrlich blauäugig. Wir müssen uns auch als grüne oder sozialdemokratische Justizministerinnen und Justizminister natürlich damit beschäftigen, daß manche Menschen diese Entwicklung nicht vollziehen. Daß sie auch nach einer langen Freiheitsstrafe nicht in der Lage sind, straffrei zu leben, außerhalb der Mauern. Und dafür müssen wir ihnen begleitende Hilfen an die Hand geben. Beispielsweise Bewährungshilfe, beispielsweise Führungsaufsicht, beispielsweise betreutes Wohnen. Also ein Entlassen nach Ablauf der Strafhaft in eine dann den Menschen auf sich gestellte (!) Freiheit kann die Sicherheit gefährden und deshalb brauchen wir da begleitende Möglichkeiten.

Moderator:

Was wollen Sie denn mit diesen Begleitungen auffangen: das, was Sie im Knast angerichtet haben an Schäden, zum Beispiel nach einem langen Aufenthalt, oder das, was derjenige mit hereinbringt - wie es immer heißt - an Sozialisationsschäden, an Defiziten?
Lütkes:

Wir haben einen Menschen, der eine Straftat begangen hat, und der eine lange Lebenserfahrung hat, also eine Persönlichkeit, die - wenn sie völlig mit sich im Reinen wäre - vielleicht nicht in diese Situation gekommen wäre. Also gibt es etwas aufzuarbeiten, zu verändern während des Strafvollzugs. Nun bin ich auch nicht so blauäugig, zu meinen, daß der Strafvollzug nur (zu Veränderungen) in diesem positiven Sinne führt, weil es natürlich auch schwierige Situationen gibt. Allein die Mehrfachbelegung, das Umgehen mit dieser völlig neuen Situation, kann möglicherweise auch zu einer Verhärtung in der Person führen, so daß der gesamte Vollzug durch den Begriff des Behandlungsvollzuges diese Persönlichkeit lenken, leiten und auch verändern möchte. Das ist natürlich das Ziel des Strafvollzuges, daß - wenn die Entlassung vor der Tür steht - wir dieses Ziel auch erreicht haben. Der Strafvollzug soll ja die Persönlichkeit nicht noch mehr schädigen, sondern sie ein bißchen verbessern.

\section{Moderator:}

Sind das Kategorien für Sie, Herr Kusch, in denen Sie auch denken und nach denen Sie handeln: die Persönlichkeit nicht noch mehr schädigen, sondern Hilfen zu geben, nach der Haftzeit ein Leben ohne Straftaten zu führen? Oder sagen Sie, das ist sozialpädagogischer Quatsch?

Kusch:

Nein, solange man sich allgemein so äußert, wie Frau Lütkes es gerade getan hat, wird sich kaum vernünftiger Widerspruch erheben können. Interessant wird es dann, wenn man sieht, wie mit einer großen Zahl von Straftätern umgegangen wird und wie oft Chancen gewährt werden und wie oft Chancen ein zweites, drittes oder viertes Mal gewährt werden. Und die Bevölkerung, selbst die, die sich nicht mit Strafvollzugsfragen so beschäftigt, daß es Experten sind, hat ein sehr feines Gespür dafür, wo staatliche Gerechtigkeit vernünftig gehandhabt wird und wo die Blauäugigkeit in ideologischen Unsinn umschlägt. Und wenn jemand fünfmal Bewährung bekommen hat, fünfmal versagt hat, fünfmal trotz Bewährungsauflagen neue Straftaten, möglicherweise schwerere als bei der Vortat begangen hat, dann ist es einfach blauäugiger Unsinn, ihm beim sechsten Mal Bewährung $\mathrm{zu}$ geben, weil es überhaupt keine Lebenserfahrung gibt, warum nun plötzlich beim sechsten Mal ein Wunder passieren soll.

\section{Moderator:}

Nehmen wir jemanden, der eine wiederholte Tat begangen hat, nach mehrmaligen Haftaufenthal- ten, nach tatsächlich vollzogener Haft. Herr Pfeiffer, passiert dann das Wunder, dadurch, daß er in Haft einsitzt, ändert sich 'was?

Pfeiffer:

Nein, die Rückfallquoten nach einer Haftzeit sind sehr, sehr hoch. Je jünger die Haft vollzogen wird, umso höher die Rückfallquote, bei UnterZwanzigjährigen ist sie dann etwa achtzig Prozent. Also keine Illusionen: Im Gefängnis werden die Wenigsten besser. Von daher ist die Entscheidung eines Richters, jemanden hinter Gitter zu bringen in einer Situation, wie Herr Kusch sie geschildert hat, nicht die Illusion, durch den Vollzug wird er jetzt ein besserer Mensch und danach wird er keine Straftaten mehr begehen, sondern schlicht angesichts der Schwere seiner Taten, angesichts der Rückfallgefahr, die er dokumentiert hat durch sein ständiges Immer-wieder-Straftaten-Begehen, müssen wir ihn aus dem Verkehr ziehen und das in Relation zu der Schwere seiner Tat. Und da haben wir überhaupt keine Debatte untereinander: Das ist richtig! Herr Kusch hat hier etwas an die Wand gemalt, als ob das irgendwo anders beurteilt würde, aber Sie finden landauf, landab, daß in einer solchen Situation dann der Richter sagt: Schluß mit den Chancen - jetzt fährt er ein!

.... Im Verlauf der Sendung ging es auch um Vollzugslockerungen, um Ausführung, Ausgang und Urlaub aus der Haft. ....

Pfeiffer:

Richtig ist, daß die Mehrheit der Gefangenen die Segnungen wie Urlaub oder ähnliches gar nicht bekommt. Ich habe gerade bei mir mal auszählen lassen: Gerade ein Prozent der Gefangenen haben im letzten Jahr Urlaub aus der Haft bekommen. Das wird in der Öffentlichkeit weit überschätzt, was da an Lockerungen geschieht, weil manche gezielt damit Angst machen und populistisch Stimmungen erzeugen wollen. Aber die Wirklichkeit ist, daß der Vollzug extrem zurückhaltend geworden ist. Früher war das noch ein bißchen anders, vor zehn, fünfzehn Jahren. Aber in den letzten zehn Jahren ist das extrem zurückgefahren worden und jetzt sind wir auf einem so niedrigen Stand von Lockerungen angekommen, daß wirklich niemand behaupten kann, wir würden da irgendwo übertreiben. Vor allem: Es gibt gar keine großen regionalen Unterschiede, von Nord 
nach Süd, von Ost nach West, ich hab' das gerade wieder überprüfen lassen. Also, da wird viel in Ideologie gemacht, in Wirklichkeit ist der Vollzug extrem zurückhaltend geworden mit solchen Lockerungen.

Ein Hörer ruft an und fordert strengere Kontrollen in den Haftanstalten, um das Einschmuggeln und den Konsum von Drogen zu unterbinden

\section{Moderator:}

Herr Kusch, Sie haben sich gerade vorgenommen in Hamburg den Drogenkonsum in den Haftanstalten weitgehend zu verhindern. Wie wollen Sie strenger kontrollieren ....?

Kusch:

Indem alle Maßnahmen, bei denen Drogen erfahrungsgemäß in Haftanstalten eingeschleust werden können, darauf überprüft werden können, ob das Maß an freiem Austausch von Waren, freier Zugang von Personen in einer verantwortbaren Relation zu den Sicherheitskontrollen ist. Wenn man die Besuche, die das Strafvollzugsgesetz vorsieht, von Angehörigen, über das Maß hinaus ausdehnt, das das Strafvollzugsgesetz vorschreibt, muß man politisch verantworten, daß die ausgedehnten Besuche natürlich bei gleicher Zahl von Vollzugsbediensteten zu redu-

Sheriff Arpaio hat bei der Bevölkerung eine außerordentlich hohe Anerkennung. Er macht geradezu offensiv Öffentlichkeitsarbeit und prahlt damit, wie schlecht es seinen Gefangenen geht. Er bekommt dafür höchstmögliche Zustimmung von Menschen, die entscheiden, wer der nächste Sheriff ist. Willensbildungsprozesse mögen einem gefallen oder auch nicht, man muß sie zur Kenntnis nehmen.

ziertem Kontrollaufwand führen. Denn ein Beamter kann pro Tag nur das leisten, was ein Vollzugsbediensteter leisten kann. Wenn er fünf Besuche kontrollieren muß, kann er lange nicht so gründlich kontrollieren wie wenn er nur einen (kontrollieren) muß. An dieser Stelle habe ich .... in Hamburg bestimmte Situationen vorgefunden, bei denen das Maß der Kontrolle nicht in vernünftiger Relation war zu dem, was man an Warenaustausch beispielsweise und an Besuchsmöglichkeiten ermöglicht.

\section{Moderator:}

Herr Pfeiffer, richtet sich das Wahrnehmen von Gefangenenrechten, also das Recht auf Besuch beispielsweise, nach den organisatorischen Möglichkeiten der Anstalt. Das heißt, hat eine Anstalt weniger Personal, dann gibt's auch weniger Besuch?
Pfeiffer:

Das ist ganz sicher ein Faktor, der da von Bedeutung ist. Man muß es ja verantworten können, wenn man Menschen hereinläßt. Und wenn man das Personal nicht hat, dann schränkt man zwangsläufig auch die Möglichkeiten der Kontakte nach draußen ein. Das ist ganz sicher so. Von daher sind wir alle darauf angewiesen, daß wir nicht nur genügend Personal haben, sondern dieses Personal auch so führen, daß sie engagiert ihre Arbeit machen. Ich mach' deshalb in diesem Jahr, weil es so entscheidend auf das Personal ankommt, eine Befragung aller Vollzugsbediensteten in Niedersachsen, wo wir von ihnen wissen wollen, was sie frustriert, was sie ärgert, wo sie uns Verbesserungsvorschläge machen, damit wir sie wirklich engagiert an der Mitarbeit im Vollzug halten können. ....

\section{Moderator:}

Frau Lütkes, zu wenig Personal und dann gibt's auch weniger Besuch - widerspricht das nicht dem Gedanken des Strafvollzugsgesetzes?

Lütkes:

Wir sind natürlich verpflichtet, die Ansprüche der Gefangenen aus dem Strafvollzugsgesetz möglichst zu erfüllen. Aber .... wir brauchen Personal, um das zu können. Deshalb - ich glaube, da sagen wir alle drei das Gleiche, gerade in der jetzigen schwierigen Haushaltssituation - gehört es zur Aufgabe eines Justizministers, einer Justizministerin, auch dafür zu arbeiten und zu sorgen, daß die Ausstattung der Justizvollzugsanstalten, die Personalausstattung nicht reduziert wird. Ich kann es nur für mich sagen, daß meine Kabinetts-Kollegen das auch verstehen, daß wir einen ganz bestimmten PersonalLevel haben müssen, einmal für die Sicherheit in den Justizvollzugsanstalten und um Besuch beispielsweise, Warenaustausch auch wirklich zu kontrollieren und die Behandlung fortzusetzen. Da ist eine klare Linie

\section{Moderator:}

Nun gibt es im Ausland Bestrebungen, den Vollzug härter zu machen. In anderen Ländern ist für Gefangene beispielsweise der Fernseh-Konsum eingeschränkt, sie leben in Massenunterkünften, sie bekommen Essen, das sie draußen nie anrühren würden, und sie müssen Anstaltskleidung tragen. Wir haben aus Arizona gehört, da tragen sie in einem Knast sogar rosa Unterwäsche. Und sie haben kein Freizeitangebot. Herr Kusch, wem nützt das?
Kusch:

Das habe ich mich in Arizona, wo ich das angeschaut habe, auch gefragt. Aber es nützt einem nichts, daß man die ganze Welt mit den Maßstäben, die wir in Deutschland lernen und hochhalten - und hochhalten müssen - bewertet. Und es dient auch unserer eigenen Selbsterkenntnis, mal festzustellen, daß in anderen Ländern erstens andere Sitten herrschen, und zweitens möglicherweise die Maßstäbe, die dort herrschen, nicht per se, wenn sie mit unseren nicht übereinstimmen, gleich mit dem Wort "menschenunwürdig « versehen werden. Deshalb hab' ich in Arizona die Anregung aufgenommen, daß die Selbstverständlichkeit, mit der Einzelvollzug in Deutschland als unumgänglich dargestellt wird, in Frage gestellt wird. Was in Arizona mich außerdem zwar befremdet hat, aber von mir zu Kenntnis zu nehmen ist: Sheriff Arpaio, der diesen abenteuerlichen Vollzug gestaltet, der genauso ablehnenswert ist, wie Sie es in Ihrer Frage gerade schon geschildert haben, hat bei der Bevölkerung eine außerordentlich hohe Anerkennung. Das heißt, er hat dort nicht einen Strafvollzug, wo hinter hohen Mauern unerklärliche Dinge passieren, sondern er macht geradezu offensiv Öffentlichkeitsarbeit. Er prahlt damit, wie schlecht es seinen Gefangenen geht und bekommt dafür höchstmögliche Zustimmung von Menschen, die entscheiden, wer der nächste Sheriff ist. Und Willensbildungsprozesse mögen einem gefallen oder auch nicht, man muß sie zur Kenntnis nehmen.

Burkhard Plemper, Bötelkamp 31,22529 Hamburg

NEUE BÜCHER

Thomas Weipert

Lebenswelt Gefängnis

Einblick in den Jugendstrafvollzug mit Berichten junger Gefangener

Centanrus 2003, 17,40€

ISBN: 3-8255-0404-2

Jede Generation muß erneut die Erfahrung machen, daß Resozialisierung im Vollzug kaum gelingt. 